\title{
PROBLEMAS DE ADHERENCIA A DIETOTERAPIA EN PACIENTES HIPERTENSOS PEHUENCHES
}

\section{PROBLEMS OF ADHERENCE TO DIET THERAPY IN HYPERTENSIVE PEHUENCHE PATIENTS}

\author{
Patricio Oliva M. (1), Kristian Buhring B. (2) \\ (1) Dirección de Investigación. Facultad de Odontología. \\ Universidad del Desarrollo. Concepción, Chile. \\ (2) Departamento de Salud Pública. Facultad de Medicina. \\ Universidad Católica de Concepción. Concepción, Chile.
}

\begin{abstract}
Background: Hypertension is a disease with a high national prevalence which increases in sectors where there are indigenous populations. The complexity of the disease lies in the behavior that must be maintained to effectively adhere to medication and diet therapy. Objective: To understand the cultural factors that are associated with problems of adherence to diet therapy in hypertensive pehuenches. Methods: Qualitative study in hypertensive patients who do not adhere to diet therapy. We interviewed 42 individuals in the municipality of Alto Bio Bio for 6 months, analyzing them using semantic structural analysis completed with the saturation point of speech. Results: Discourse analysis showed the following results: a) ethnic foods have a symbolic value, b) ethnic foods are described as "natural" in opposition to any food that does not occur in their environment, although they are based in a high consumption of fats, proteins and carbohydrates, c) nonexistent biochemical properties ascribed to ethnic foods justify their use. Conclusion: there are cultural differences between the prescription of the nutritionist and the eating behavior of pehuenches, which affect non-compliance with diet and disease complications.
\end{abstract}

Key words: Adherence to diet therapy, hypertension, Pehuenche.

Este trabajo fue recibido el 1 de Marzo de 2011 y aceptado para ser publicado el 18 de Mayo de 2011.

\section{INTRODUCCIÓN}

Las enfermedades crónicas no transmisibles constituyen la principal causa de muerte en gran número de países desarrollados (1), en Chile no sólo poseen una alta prevalencia, sino que además están en aumento (2), generando un problema de salud pública, además de una fuerte carga económica al sistema sanitario.

El problema radica en que el control de este tipo de enfermedad requiere un cambio conductual de los sujetos que permita generar adherencia a tratamientos farmacológicos y especialmente dietoterápicos. La dietoterapia puede ser altamente efectiva para el tratamiento de enfermedades crónicas (3), pero requieren de la participación de los sujetos, adoptando conductas alimentarias saludables. El problema del cumplimiento dietoterápico en pacientes crónicos hipertensos se describe en otros estudios como un factor de riesgo de morbimortalidad a largo plazo (4). Las investigaciones culturales sobre el cumplimiento de dietoterapias se han centrado principalmente en el soporte social del paciente, comportamiento y educación (5), aunque se han logrado identificar más variables socioculturales asociadas (6).

Epidemiológicamente las tasas de enfermedades crónicas poseen una prevalencia mayor en comunidades indígenas de Chile (7), principalmente por los determinantes de salud asociado a las características históricas culturales (8), a lo que se le suma su condición de ruralidad que proporciona un mayor riesgo en enfermedades crónicas (9). La hipertensión arterial, particularmente, posee tasas de prevalencia de $30,1 \%$ con un sostenido aumento anual (10), con 27,\% en población mapuche (11), existiendo problemas de cumplimiento farmacológico (12) y dietoterápico (6) aún cuando es conocido el efecto negativo de mantener una dieta inadecuada cuando se 
padece dicha enfermedad (13). Los mapuches en Chile representan alrededor del $10 \%$ de la población nacional y aproximadamente un $0,5 \%$ representa a la población Pehuenche (mapuches que habitan exclusivamente a las orillas del río Biobío en la zona cordillerana de los Andes de la VIII y IX Región), culturalmente comparten la misma historia y prácticas cotidianas.

Las conductas no adherentes de los pehuenches en torno a la hipertensión arterial derivan en el no reconocimiento de esta como tal (10) y además se contraponen con sus propias concepciones sociales de enfermedad (6) por lo cual resulta altamente complejo la incorporación de tratamientos que intenten modificar sus conductas por ello validadas y que poseen un alto valor simbólico (14), centrándose en alimentos que tienen a su alcance (15).

La importancia del estudio radica en la comprensión efectiva de los factores que influyen en la adherencia a tratamientos dietoterápicos en población hipertensa de una cultura que no define a las enfermedades crónicas como propias. La combinación de elementos que dificultan el cumplimiento dietoterapéutico en esa población particular entrega información valiosa sobre las variables que componen el comportamiento alimenticio de ellos. Por lo cual el objetivo de esta investigación fue establecer los elementos socioculturales que influyen en la adherencia a la dietoterapia en pehuenches hipertensos.

\section{SUJETOS Y MÉTODOS}

El diseño utilizado fue cualitativo - etnometodológico, centrándose en la interpretación de la realidad por medio del discurso (16) sobre la salud de los pehuenches, especialmente todo los vinculados a conceptos y comportamientos que los individuos realizan de su vida cotidiana (17). El diseño cualitativo a pesar de ser incipiente, es utilizado comúnmente en investigaciones en nutrición (18) y tiene como fin en la presente investigación facilitar la interpretación de la integración de los conceptos intersubjetivos relacionados con la adherencia dietoterápica.

El universo para este estudio fueron los pacientes pehueches hipertensos diagnosticados medicamente en sus respectivos centros de salud, pero que no asistían a controles nutricional desde hace 6 meses, en la comuna de Alto Bio Bio.

Los criterios de selección de sujetos para el estudio fueron: a) pehuenches mayores de 18 años, b) pehuenches atendidos por el nutricionista en el Centro de Salud Familiar (CESFAM) de la comuna investigada, c) pehuenches con Hipertensión Arterial Primaria diagnosticada d) pehuenches con prescripción de tratamiento dietoterapéutico que no adhieran a dicha terapia. Se reclutó a los sujetos mediante informantes claves pe- huenches que conocían la condición de sus pares. Dada la orientación de la investigación, y de acuerdo con el problema que se planteó, no se consideró necesario investigar sobre el discurso y las conceptualizaciones que derivan ello en pacientes en población adherente, debido a que dichos sujetos se encontraban integrados al sistema sanitario "occidental" que al tradicional pehuenche, llegando a un total de 42 individuos entrevistados (20 hombres, 22 mujeres, con un promedio de 48 años de edad)de un universo de 210 pacientes diagnosticados (19). Como investigación cualitativa se articula en base los conceptos, enfatizando en la profundidad de la investigación más que en la cantidad, a pesar de que la investigación se efectúa a una quinta parte de la población con problemas dietoterápicos.

El diseño muestral se establece bajo el paradigma cualitativo seleccionado, escogiéndose el muestreo de casos voluntarios (20), que consiste en la participación voluntaria de individuos que representen fielmente tanto la cultura pehuenche como los criterios de selección enunciados.

La recolección de la información se realizó mediante entrevistas en profundidad, siendo el instrumento más apropiado para la recolección, debido a lo flexible que puede ser al momento de enfrentarse a la información emitida por los entrevistados. El procedimiento de recolección de información implicó entrevistar a individuos que cumplían con los criterios de selección hasta llegar al punto de saturación de la información, en el cual se concluye la investigación producto de la reiteración de los conceptos presentes en los discursos de los entrevistados. Se ejecutó un procedimiento de transcripción de entrevistas, realizando un análisis de contenido de entrevista por entrevista.

Debido a que la metodología cualitativa trabaja con interpretación del lenguaje (21), la información se evaluó mediante análisis semántico estructural. Este análisis se basa en la generación de categorías lingüísticas que surgen a partir del discurso de los entrevistados, concentrándose en los conceptos que responden al problema y omitiendo aquello que no tiene directa relación con éste. El análisis logra objetivizar el discurso mediante la interpretación sistemática del tex to generando nuevas construcciones y explicaciones sobre la problemática a estudiar (22).

\section{RESULTADOS}

Los pehuenches hipertensos crónicos no sólo presentan problemas con la adherencia farmacológica sino además poseen una relación especial con la alimentación basándose en su estructura histórica y cultural. Esta dieta se centra en el consumo de carne, grasas y carbohidratos, 
especialmente porque es aquello que consiguen en su entorno con mayor facilidad, poseyendo una vinculación histórica asociada a lo natural y "sano" profundamente establecida en las prácticas cotidianas.

Los pehuenches bajo tal premisa realizan una comparación permanente entre la "dieta étnica" y la "dieta occidental", puntos de comparación que determinan la apropiación de un estilo de alimentación en contraposición de otro tipo de dieta que es asociada a elementos ajenos y por lo tanto, altamente cuestionados. Se establece con ello dimensiones categorizadas de acuerdo a los conceptos que derivan del análisis, facilitando la redacción y la evaluación de los resultados, que se expresan en las siguientes categorías:

1) Poseen un valor simbólico, esto quiere decir que se encuentran ligados a procesos religiosos y sociales de gran valor cultural, por ejemplo el uso del piñón como base de la dieta pehuenche: “... uno tiene que comer cosas que siempre hemos comido porque así mantenemos lo que nos dejaron nuestros padres..." (entrevistado n $\left.{ }^{\circ} 4\right)$ donde la tradición es fundamental para la incorporación de nuevos alimentos a la dieta: “... nuestros padres comieron como nosotros, y fueron sanos, no tenemos porqué cambiar las cosas porque nos dicen los expertos de afuera... (entrevistado $n^{\circ} 6$ ).

El valor simbólico conlleva a que los sujetos definan los alimentos autóctonos como de mejor calidad, debido a que están en contacto directo con la producción de estos: poseen una pequeña agricultura de subsistencia y ganadería, se vinculan con el proceso y este acto garantiza la calidad de los productos generados. Ejemplo: “... uno sabe que siembra... es mucho mejor porqué uno conoce lo que está comiendo..."(entrevistado $n^{\circ} 3$ ), por lo cual una dieta correcta desde la perspectiva de los entrevistados debe integrar adecuadamente los alimentos conocidos. Por otro lado el simbolismo está relacionado con sus ceremonias religiosas y médicas, siendo el personaje de la machi o la hierbatera quien recomienda la alimentación étnica como fundamental para mantener las tradiciones, la incorporación de dietas que no están consideradas como propias se ven limitadas en dimensiones que van desde lo político hasta lo religioso: “... la hierbatera siempre dice que hay que comer lo de nuestros padres... sino todo se acaba... por algo se empieza y es fácil caer comer cosas y perder las tradiciones..."(entrevistado $\mathrm{n}^{\circ} 23$ )

2) Las dietas propias son catalogadas como naturales, en contraposición a dietas que se denominan "occidentales" ejemplificados en la comida chatarra. Esta clasificación se apoya en el hecho de que son los propios pehuenches quienes mantienen sin alteración (uso de fertilizantes o alimento procesado para animales) los animales y cosechas de las cuales se alimentan. La variedad resulta restringida pero suficiente para satisfacer sus necesidades dietéticas, por ejemplo se argumenta que: “...uno come sano, ve lo que está comiendo, no tiene ningún químico ni cosas que no son naturales como en la cuidad..." (entrevistado ${ }^{\circ} 11$ ), lo cual impide la modificación de la conducta alimentaria en una enfermedad crónica, debido a las características de largo plazo de estas: “... el nutricionista de la posta quiere que coma cosas que ni conozco... yo comeré cosas sana y limpias y lo que mis padres comieron, además ni siquiera me siento enfermo..." (entrevistado $\left.\mathrm{n}^{\circ} 15\right)$.

3) Se le asignan propiedades bioquímicas en los alimentos presentes en la dieta pehuenche: en el discurso pehuenche en tema alimentario se le entrega propiedades bioquímicas a los alimentos de forma arbitraria pero siempre favorable a sus dietas: “... el cordero y la infundía tiene mucha vitamina $\mathrm{C}$ que sirve para el embarazo y para que crezcan fuertes los críos..." (entrevistado $\left.\mathrm{n}^{\circ} 12\right)$, “...el cerdo tiene calcio y con las hierbas que le ponemos sirve para que los enfermos se sientan más fuertes..." (entrevistado $n^{\circ} 7$ ). Por lo cual se presenta un proceso de hibridación cultural que afecta a la dieta, generando un sistema de conocimiento que justifica al paciente enfermo crónico no adherente a la dietoterapia.

Los argumentos dificultan la implementación de dietoterapia por parte del nutricionista en pacientes enfermos crónicos debido a la alta valoración que tienen de sus costumbres alimentarias y de la no aceptación de las dietas "occidentales".

\section{DISCUSIÓN}

El territorio de Alto Bio Bío presenta una situación intercultural en salud que genera un escenario de interacción donde los actores étnicos establecen distinciones culturalmente particulares, especialmente por la dualidad que se expresa por un lado por pehuenches que acuden a los centros de atención de medicina occidental -hospitales, centros de salud familiar, postas rurales, y estaciones médico rural- $\mathrm{y}$ asisten también a los agentes médicos indígenas.

Por otro lado existe la coexistencia de hierbas y expresiones culturales - tradicionales pehuenches de diagnóstico y tratamiento, que posee un importante sustrato mágico religioso y otras formas relativas al conocimiento médico concebido como ciencia biológica, expresada en este caso en la dietoterapia (23), enmarcándose en el concepto de "hibridación cultural", que permite que los individuos validen de manera homogénea elementos de ambas culturas como si fueran una sola (24).

En el contexto cotidiano pehuenche existe una constante contraposición de dietas étnicas y occidentales, 
la dietoterapia resulta irruptiva, compleja y disocia al pehuenche de lo que él considera su historia.

El impacto que posee esta dicotomía en estas comunidades deriva en complicaciones de salud pública a nivel nacional, debido a que la no adherencia al tratamiento dietoterápico eleva los índices de enfermedades asociadas y sus complicaciones, siendo las políticas sanitarias incompletas si no incorporan la visión étnica de la comprensión de la enfermedad, dificultando el enfoque preventivo que los equipos de salud quieren asumir para la disminución de las enfermedades crónicas (25).

El estudio presenta interrogantes sobre la forma de abordar nutricionalmente las enfermedades crónicas en poblaciones que poseen una cultura con una epidemiología diferente a la occidental (el modelo etiológico atribuye la enfermedad a fuerzas espirituales operando con una lógica moral y social, donde la enfermedad aparece cuando el enfermo trasgrede de alguna regla social), en la cual la comparación permanente que el paciente pehuenche realiza resulta desfavorable para la adopción de la dietoterapia como elemento permanente para el control de la hipertensión.

Los resultados son relevantes en establecer una línea de base para posteriores estudios sobre conductas nutricionales de los pehuenches y generar una correcta estrategia de tratamiento dietoterápico en este tipo de población. Para una próxima investigación sobre la temática se sugiere la incorporación de información cuantitativa y con ello facilitar la triangulación de la información, y no sólo tener profundidad en los datos, sino que también posea la capacidad de ser extrapolada a poblaciones mayores.

\section{RESUMEN}

Introducción: La hipertensión arterial es una patología con una alta prevalencia nacional que aumenta en sectores con poblaciones indígenas. La complejidad de la enfermedad radica en la conducta que se debe mantener para adherir eficazmente al tratamiento farmacológico y dietoterápico. Objetivo: Comprender los factores culturales que se asocian a los problemas de adherencia a la dietoterapia en pacientes hipertensos pehuenches. Metodología: Estudio cualitativo, en pacientes hipertensos pehuenches que no adhieren a Dietoterapias. Se entrevistó a 42 pehuenches de la Comuna de Alto Bio Bío durante 6 meses. El análisis de los datos se realizó mediante análisis semántico estructural. La muestra se logró mediante el punto de saturación. Resultados: El análisis de discurso arroja los siguientes resultados: a) los alimentos étnicos tienen un valor simbólico b) los alimentos étnicos son calificados como "naturales" en contraposición a cualquier alimento que no se produzca en su medio, aunque se basan en un alto consumo de grasas, proteínas y carbohidratos, c) a los alimentos étnicos se les atribuye propiedades bioquímicas inexistentes con los cuales se justifica su consumo. Conclusión: Existen diferencias culturales entre la prescripción del nutricionista y lo realizado por pehuenches que inciden en el no cumplimiento de la dietoterapia y complicación de la enfermedad.

Palabras clave: Adherencia a dietoterapia, hipertensión arterial, Pehuenches.

Dirigir la correspondencia a:

Profesor

Patricio Oliva M.

Universidad del Desarrollo, Concepción

Dirección de Investigación

Barros Arana 1734, Concepción. Chile.

Fono: 041-2268544

Fax: 56-41-2268501

Email: patricioolivamella@gmail.com

\section{BIBLIOGRAFÍA}

1. Organización Mundial de la Salud. Prevención de las enfermedades crónicas. Una inversión vital. Panorama general. 2005. pp. 1 -3.

2. Fagalde M., Solar J., Guerrero M. et al. Factores de riesgo de enfermedades crónicas no transmisibles en funcionarios de una empresa de servicios financieros de la Región Metropolitana. Rev Méd Chil 2005;133(8):919-28.

3. Troncoso P Claudia, Sotomayor C Mauricio, Ruiz H Fernanda, Zúñiga T Carla. Interpretación de los conocimientos que influyen en la adherencia a la dietoterapia en adultos mayores con Diabetes Tipo 2 de una comuna rural. Rev Chil Nutr 2008; 35(4): 421-6.

4. Ramírez M. Estrategia mundial sobre régimen alimentario, actividad física y salud. INCAP Instituto de Nutrición de Centro América y Panamá. 2004.

5. Roman A, Oscar, Valenzuela, María A, Badilla S, Marta et Al. Optimización de la reducción de la presión arterial en hipertensos esenciales. Rev Méd Chil 2002; 130(5): 519 - 26.

6. Amigo I, Fernández A, González A y Herrera J. Relajación muscular y monitorización ambulatoria de la presión arterial en la hipertensión arterial. Psicothema 2002; 14: 48 - 52.

7. Diagnóstico de la situación en salud de las comunidades pehuenches de Callaqui y Cauñicu. "Apoyo al Diseño de Experiencias Piloto en Modelo de Salud Intercultural en las Comunidades Pehuenche de la Comuna de Alto Bio Bío, Octava Región, Provincia 
del Bio Bío” Servicio de Salud. Bio Bío. Año 2005.

8. Mora, Ziley. "Estudio de Sistematización en Terreno de las Experiencias de Salud Intercultural en el Área de Desarrollo Indígena de Alto Bio Bío. Impacto y presencia del sistema de salud en las comunidades indígenas Pehuenches. Evaluación de programas, experiencias, dificultades, aciertos, catastros y propuestas sistémicas para un posible modelo de salud intercultural en Alto Bio Bío.”. Los Ángeles, Chile, Diciembre 2002.

9. Larenas G, Arias G, Espinoza O, Charles M, Landaeta O, Villanueva S Et Al. Prevalencia de diabetes mellitus en una comunidad mapuche de la IX Región, Chile. Rev Méd Chil 1985; 113: 1121-5.

10. Pérez B Francisco, Carrasco P Elena, Santos José Luis, Calvillán Marcelo, Albala B Cecilia. Prevalencia de obesidad, hipertensión arterial y dislipidemia en grupos aborígenes rurales de Chile. Rev Méd Chil 1999; 127(10): 1174.

11. Carrasco P Elena, Pérez B Francisco, Angel B Bárbara, Albala B Cecilia, Santos M J Luis, Larenas Y Gladys et al. Prevalencia de diabetes tipo 2 y obesidad en dos poblaciones aborígenes de Chile en ambiente urbano. Rev Méd Chil 2004; 132(10): 1192.

12. Representaciones sociales de salud y adherencia farmacológica antihipertensiva en población pehuenche. Oliva, P. Narváez C. Rev Chil Salud Pública 2009; 13(2): $69-71$.

13. Esquivel Solís, Viviana. Jiménez Fernández Maristela. Aspectos nutricionales en la prevención y tratamiento de la hipertensión arterial. Rev Costarriscense Salud Pública 2010; 19: 42 - 4.

14. Jerome N, Kandel R, Pelto G: Nutritional anthropology: contemporary approaches to diet and culture. New York. Redgrave Publishing Company, 1980.

15. Rosique G Javier, Restrepo C María Teresa, Manjarrés C Luz Mariela, Gálvez A Aida, Santa M Johana. Estado nutricional y hábitos alimentarios en indígenas Embera de Colombia. Rev Chil Nutr 2010; 37(3): 270-80

16. Schutz, Alfred. El problema de la realidad social. $2^{\circ}$ edición Argentina: Amorrotu edit. 1995. p 115.

17. Oliva M Patricio, Buhring B Kristian, Godoy T Sofía, Bustos T María. Percepción de la función profesional del nutricionista por parte de los usuarios de Atención Primaria. Rev Chil Nutr 2010; 37(2): $165-8$

18. Gil Nebot $\mathrm{M}^{\mathrm{a}}$ Ángeles, Estrada Ballesteros Carmen, Pires Alcalde $\mathrm{M}^{\mathrm{a}}$ Luisa, Aguirre Martín-Gil Ramón. La investigación cualitativa y la promoción de la salud en la Comunidad de Madrid. Rev Española
Salud Pública 2002; 76(5): 453.

19. Ilustre Municipalidad de Alto Bio Bío. Plan Comunal de Salud. 2006.

20. Hernández Sampieri, Roberto. Metodología de la investigación. $4^{\circ}$ edición México: Mc Graw Hill. 2003. p. 566.

21. Remy J. "Métodos de análisis de contenido de Sociología”. Bruselas, Sain Louis University edit., 1991. p 79.

22. Pérez Andrés Cristina. ¿Deben estar las técnicas de consenso incluidas entre las técnicas de investigación cualitativa?. Rev Española Salud Pública 2000; 74(4):78.

23. Crocker Sagastume René, Cosío González Antonio, López López Martina, Ruiz Domínguez Liah, Andrade Ureña Diana, Gutiérrez Gómez Yareni. Interculturalidad alimentario-nutricional en la etnia Wixarika de México. Rev Española Salud Pública 2004; 78(6):691 - 700.

24. Jiménez-Ottalengo, R. Y Salas Gómez, L. E. Una experiencia de educación alimentaria en el Rincón Zapoteca. Cuad Nutr Alim México 1998; 21(3):5.

25. Castillo C Álvaro, Kain B Juliana. Consejería en vida sana y cambio de conductas en escolares obesos: Intervención controlada en madres/Cuidadoras. Rev Chil Nutr 2010; 37(2): 155 - 63. 